\title{
SQUARE FUNCTION ESTIMATES AND THE $T(b)$ THEOREM
}

\author{
STEPHEN SEMMES
}

(Communicated by J. Marshall Ash)

\begin{abstract}
A very simple proof is given of the sort of square function estimates provided by the $T(b)$ theorem. The approach is based on the bilinear methods of Coifman and Meyer. Among the applications is an easy proof of the boundedness of the Cauchy integral operator on Lipschitz graphs.
\end{abstract}

The $T(b)$ Theorem [MM, DJS] gives a criterion for a singular integral operator $T$ to be bounded on $L^{2}$. It also gives a method for proving certain types of square function estimates. In this paper we give a simple new approach to this result on square functions. We do not know how to use methods of this type to derive the full $T(b)$ Theorem, but we can recover a number of its applications, in particular the boundedness of the Cauchy integral on Lipschitz graphs.

The general setup is as follows. Suppose we are given a family of functions $\theta_{t}(x, y), t>0, x, y \in \mathbf{R}^{n}$, such that for some $C, \alpha>0$,

$$
\begin{gathered}
\theta_{t}(x, y)=0 \quad \text { if }|x-y| \geq C t, \\
\left|\theta_{t}(x, y)-\theta_{t}\left(x, y^{\prime}\right)\right| \leq C t^{-n-\alpha}\left|y-y^{\prime}\right|^{\alpha},
\end{gathered}
$$

for all $t>0, x, y, y^{\prime} \in \mathbf{R}^{n}$. (We restrict ourselves to $\theta_{t}$ 's that are compactly supported only for notational convenience. We could as easily work with the case where $\theta_{t}(x, y)$ has adequate decay as $|x-y| \rightarrow \infty$.) We want to find conditions under which we have the square function estimate

$$
\int_{0}^{\infty}\left\|\Theta_{t} f\right\|_{2}^{2} \frac{d t}{t} \leq C\|f\|_{2}^{2},
$$

where

$$
\Theta_{t} f(x)=\int \theta_{t}(x, y) f(y) d y .
$$

The estimate (3) should hold if we have some cancellation on $\theta_{t}$. For example, it is well known that (3) holds if $\int \theta_{t}(x, y) d y=0$ for all $x, t$. (Sketch

Received by the editors July 10, 1989 and, in revised form, October 2, 1989.

1980 Mathematics Subject Classification (1985 Revision). Primary 42B20.

The author is partially supported by the National Science Foundation and the Alfred P. Sloan Foundation. 
of proof: Simple calculations show that it suffices to prove that

$$
\sup _{s} \int_{0}^{\infty}\left\|\Theta_{s} \Theta_{t}^{*}\right\| \frac{d t}{t}<\infty
$$

and

$$
\sup _{t} \int_{0}^{\infty}\left\|\Theta_{s} \Theta_{t}^{*}\right\| \frac{d s}{s}<\infty,
$$

where $\|T\|$ denotes the $L^{2}$-operator norm of $T$. These estimates are easily obtained from (1), (2), and the cancellation condition on $\theta_{t}$.)

We want to generalize this cancellation condition. Suppose $b \in L^{\infty}\left(\mathbf{R}^{n}\right)$ satisfies $|b| \geq \delta$ for some $\delta>0$.

Theorem. (3) holds if $\Theta_{t}(b)=0$ for all $t$ and if $b$ is para-accretive.

Para-accretivity is a condition that forces $b$ not to oscillate too much. For example, if $b$ is accretive-i.e., $\operatorname{Re}(b) \geq \delta$ for some $\delta>0$-then $b$ is paraaccretive. A good example of a $b$ which is not para-accretive is $b(x)=e^{i x}$ on R.

The precise definition of para-accretivity is the following. We require that there exist $\delta, \eta, C>0$ so that for each $x \in \mathbf{R}^{n}$ and $t>0$ there is a function $\varphi(y)$ on $\mathbf{R}^{n}$ satisfying

$$
\begin{gathered}
\left|\int b(y) \varphi(y) d y\right|>\delta ; \\
\operatorname{supp} \varphi \subseteq B(x, t)=\{y:|x-y| \leq t\} ; \\
\left|\varphi(y)-\varphi\left(y^{\prime}\right)\right| \leq C t^{-n-\eta}\left|y-y^{\prime}\right|^{\eta}
\end{gathered}
$$

One can derive this theorem from the methods of [DJS]. We shall instead derive it using $P_{t}-Q_{t}$ techniques of [CM].

The inspiration of our approach is a proof of this result of $[\mathrm{J}]$ in the special case where

$$
\theta_{t}(x, y)=t(x-y+i(A(x)-A(y))+i t)^{-2}
$$

with $A$ a Lipschitz function on $\mathbf{R}$. Roughly speaking, Jones showed that this $\theta_{t}$ could be written as a sum of two pieces, the first of which is essentially the same as the above but with $A=0$, and an error term that is controlled using maximal functions and a Carleson measure. He obtained this decomposition using the explicit form of $\theta_{t}$ and a lemma showing how well $A$ can be approximated by affine functions on a given interval.

We shall obtain the theorem using a decomposition of $\theta_{t}$ that is similar to Jones's, but which is abstract.

Let $P_{t}$ be any nice (one-sided) approximation to the identity on $\mathbf{R}^{n}$ :

$$
P_{t} f(x)=\int p_{t}(x, y) f(y) d y,
$$


where $p_{t}(x, y)$ satisfies $(1),(2)$, and

$$
\int p_{t}(x, y) d y=1 \text { for all } x \in \mathbf{R}^{n}, t>0 .
$$

The decomposition in our case is given by

$$
\Theta_{t}=\left(\Theta_{t}-\Theta_{t}(1) P_{t}\right)+\Theta_{t}(1) P_{t} .
$$

Here we will let $\Theta_{t}(1)$ denote also the operator of multiplication by itself.

It is easy to see that (3) holds when $\Theta_{t}$ is replaced by $\Theta_{t}-\Theta_{t}(1) P_{t}$, because this last operator sends 1 to 0 , which is the simple case we discussed before. To control the remaining term, we must show that

$$
\left|\Theta_{t}(1)(x)\right|^{2} d x \frac{d t}{t}
$$

is a Carleson measure.

By assumption, we have $\Theta_{t}(b)=0$. Hence,

$$
0=\Theta_{t}(b)=\Theta_{t}\left(\left(I-P_{t}\right) b\right)+\Theta_{t}\left(P_{t}(b)\right) .
$$

The kernel of $\Theta_{t}\left(I-P_{t}\right)$ satisfies (1) and (2), and this operator sends 1 to 0 . Hence (3) holds with $\Theta_{t}$ replaced by $\Theta_{t}\left(I-P_{t}\right)$, and standard reasoning proves that

$$
\left|\Theta_{t}\left(\left(I-P_{t}\right)(b)\right)(x)\right|^{2} \frac{d x d t}{t}
$$

is a Carleson measure.

It remains to relate $\Theta_{t}(1)$ to $\Theta_{t}\left(P_{t}(b)\right)$. We have that

$$
\Theta_{t}\left(P_{t}(b)\right)(x)=P_{t}(b)(x) \Theta_{t}(1)(x)+\Theta_{t}\left(P_{t}(b)(\cdot)-P_{t}(b)(x)\right)(x) .
$$

This last term satisfies a Carleson measure estimate because $\beta(x, t)$ does, where

$$
\beta(x, t)=\frac{1}{t^{n}} \int_{|x-y| \leq C t}\left|P_{t}(b)(x)-P_{t}(b)(y)\right| d y .
$$

The desired estimate for $\beta(x, t)$ holds because (3) holds when $\Theta_{t}$ is replaced by the operator defined by $f \mapsto P_{t} f(x)-P_{t} f(x-u t)$, for any $u,|u| \leq C$.

We conclude from $(8),(9)$, and the estimates above that

$$
\left|P_{t}(b)(x) \Theta_{t}(1)(x)\right|^{2} \frac{d x d t}{t}
$$

is a Carleson measure. It remains to get rid of the $P_{t}(b)$.

Notice that if $b$ were accretive and the kernel of $P_{t}$ positive, then we could get rid of the $P_{t}(b)$ trivially.

In the general case we have to do a little more work. The main point is that the para-accretivity of $b$ implies that the set of $(x, t)$ such that $P_{t} b(x)$ is small is well under control; it satisfies a Carleson measure condition, so that its contribution to (10) is controlled by the fact that $\left|\Theta_{t}(1)\right| \leq C$. 
There are a number of ways to do this rigorously. Here is one. The definition of para-accretivity implies that there is an operator $R_{t}$ with kernel $r_{t}(x, y)$ that satisfies (1), (2), and $\left|R_{t} b\right| \geq \delta$. Calculations just like (8) and (9) imply that

$$
\left|R_{t}(b)(x)-R_{t}(1)(x) P_{t}(b)(x)\right|^{2} \frac{d x d t}{t}
$$

is a Carleson measure. Since $\left|R_{t}(1)\right| \in L^{\infty}$, we conclude that if $\Omega=\{(x, t)$ : $\left.\left|P_{t} b(x)\right| \leq a \delta\right\}, a=\frac{1}{2}\left(\sup _{t}\left|R_{t}(1)\right|\right)^{-1}$, then

$$
\chi_{\Omega}(x, t) \frac{d x d t}{t}
$$

is a Carleson measure. This combined with (10) and $\left|\Theta_{1}(1)\right| \leq C$ imply $\left(7 \frac{1}{2}\right)$, as desired. This proves the theorem.

Before discussing applications of this, let us indicate some easy extensions.

(i) The square function $\left(\int_{0}^{\infty}\left|\Theta_{t} f\right|^{2} \frac{d t}{t}\right)^{1 / 2}$ is bounded on $L^{p}, 1<p<\infty$.

(ii) The theorem and its proof go over to spaces of homogeneous type.

(iii) Everything is essentially the same when we work with functions that take values in some finite-dimensional algebra. The main differences are that you have to be careful how you write the proof, because of noncommutativity, and condition (4) should be replaced by

$$
\left\|\left(\int b(y) \varphi(y) d y\right)^{-1}\right\| \leq C .
$$

In contrast with [DJS], we do not need extra structure on our algebra; e.g., we do not have to restrict ourselves to functions $b$ that take values in the linear span of the generators of a Clifford algebra.

Let me now describe some applications of the theorem.

Let $A: \mathbf{R} \rightarrow \mathbf{R}$ be Lipschitz, and define

$$
\theta_{t}(x, y)=t(x-y+i(A(x)-A(y))+i t)^{-2} .
$$

We can apply the theorem (with $b=1+i A^{\prime}$ ) to get (3) for this $\theta_{t}$.

A simple formal argument can be used to go from this square function estimate to the boundedness of the Cauchy integral on Lipschitz graphs. Set

$$
C(f)(x+i t)=\int_{-\infty}^{\infty} \frac{1+i A^{\prime}(y)}{x-y+i(A(x)-A(y))+i t} f(y) d y .
$$

It is not hard to see that

$$
\begin{aligned}
C_{+} f(x) & =\lim _{t \rightarrow 0^{+}} C(f)(x+i t) \\
& =\int_{0}^{\infty} t^{2}\left(\frac{d}{d t}\right)^{2} C(f)(x+2 i t) \frac{d t}{t} \\
& =\int_{0}^{\infty}\left(\Theta_{t} M \Theta_{t} M f\right)(x) \frac{d t}{t} .
\end{aligned}
$$


(I have dropped some inessential multiplicative constants in these equations. Here $M$ denotes the operator of multiplication by $1+i A^{\prime}$. The first equality uses the fact that

$$
t \frac{d}{d t} C(f)(x+i t)=\Theta_{t} M f(x) \rightarrow 0
$$

as $t \rightarrow 0$, which is proved using standard techniques. The second equality uses the Cauchy formula for derivatives.)

Thus,

$$
\int_{\mathbf{R}} C_{+} f(x) g(x) d x=\int_{\mathbf{R}} \int_{0}^{\infty}\left(M \Theta_{t} M f\right)(x)\left(\Theta_{t} g(x)\right) \frac{d t}{t} d x .
$$

The boundedness of $C_{+}$on $L^{2}$ now follows from the theorem.

This same argument works in other cases where you have so much algebra as, for example, the functional calculus problem considered in [DJS].

The next application concerns wavelets. Let us work on $\mathbf{R}$ for notational convenience. Let $a$ be a bounded accretive function on $\mathbf{R}$, so that $\operatorname{Re} a \geq \delta>$ 0 , and define $\langle f, g\rangle_{a}$ by

$$
\langle f, g\rangle_{a}=\int_{\mathbf{R}} f(x) g(x) a(x) d z .
$$

Let $\Delta$ denote the collection of dyadic intervals on $\mathbf{R}$. Y. Meyer constructed a family of functions $\psi_{I}, I \in \Delta$ on $\mathbf{R}$ that are orthonormal with respect to $\langle,\rangle_{a}$, i.e.,

$$
\begin{aligned}
\left\langle\psi_{I}, \psi_{J}\right\rangle_{a}=1 & \text { if } I=J \\
=0 & \text { if } I \neq J,
\end{aligned}
$$

and such that each $\psi_{I}$ has nice localization and smoothness properties. Formally, we have the reproducing formula

$$
f=\sum_{I}\left\langle f, \psi_{I}\right\rangle_{a} \psi_{I}
$$

but to make this rigorous and get the right sort of estimates we need to know that

$$
\|f\|_{2} \approx\left(\sum\left|\left\langle f, \psi_{I}\right\rangle_{a}\right|^{2}\right)^{1 / 2} .
$$

Meyer obtained this from the $T(b)$ theorem.

Conversely, once you have such a basis, it is not hard to obtain the $T(b)$ theorem in this context, as well as the boundedness of the Cauchy integral on Lipschitz graphs in particular.

Thus it was a natural problem to try to prove (12) directly, without using the $T(b)$ Theorem. This was accomplished by $\mathrm{P}$. Tchamitchian.

We can also obtain (12) from the theorem. The $\psi_{I}$ 's satisfy $\int \psi_{I}(x) a(x) d x$ $=0$, and they have adequate localization and smoothness to allow us to conclude that

$$
\sum\left|\left\langle f, \psi_{I}\right\rangle_{a}\right|^{2} \leq C\|f\|_{2}^{2}
$$


(The $\psi_{I}$ 's are not compactly supported, but they are good enough.) The reverse inequality can be obtained from a standard polarization argument.

It is interesting to compare this approach of Meyer-Tchamitchian to the one in [DJS]. In [DJS], the analogue of (12) was not nearly as hard as finding a version of (11), while in this wavelet approach, (11) was built into the algebra.

As our third application we derive the $T(b)$ theorem in the special case that $T^{t} 1=0, T(b)=0$. (This is the situation for $T=(D a D)^{1 / 2} D^{-1}$, where $a$ is a bounded accretive function on $\mathbf{R}$, as in [CMM].) Let $\psi(x)$ be a radial test function, not identically zero, that satisfies $\int \psi=0$. Set

$$
\psi_{t}(x)=\frac{1}{t^{n}} \psi\left(\frac{x}{t}\right), \quad Q_{t} f=\psi_{t} * f .
$$

Thus

$$
\int_{0}^{\infty} Q_{t}^{2} \frac{d t}{t}=c I \text { for some } c \neq 0
$$

so that

$$
T f=\int_{0}^{\infty} Q_{t}\left(Q_{t} T f\right) \frac{d t}{t} .
$$

One can show that the theorem applies to $\Theta_{t}=Q_{t} T$. (We do not have the compact support condition in this case, but the kernel has adequate decay.) The square function estimates for $Q_{t}$ and $\Theta_{t}$, together with (13), allow one to conclude the boundedness of $T$ on $L^{2}$.

Finally, the square function estimates for the Cauchy integral on certain hypersurfaces in $\mathbf{R}^{n}$ obtained in [S] using the $T(b)$ theorem can also be obtained using the simple argument above. In a separate paper these square function estimates will be used to obtain a lot of information about these surfaces.

\section{REFERENCES}

[CM] R. Coifman and Y. Meyer, Au-delà des opérateurs pseudo-différentials, Astérisque 57 (1978).

[CMM] R. Coifman, A. McIntosh, and Y. Meyer, L'integrale de Cauchy définit un opérateur borné sur $L^{2}$ pour les courbes Lipschitziennes, Ann. Math. 116 (1982), 361-388.

[DJS] G. David, J. L. Journé, and S. Semmes, Operateurs de Calderón-Zygmund, fontions paraaccretives et interpolation, Revista Mat. Iberoamericana 1 (1985), 1-56.

[MM] A. McIntosh and Y. Meyer, Algèbres d'opérateurs définis par des intégrales singuliéres, Comptes Rendus Acad. Sci. Paris 301 (1985), 395-397.

[J] P. Jones, Square functions, Cauchy integrals, analytic capacity, and harmonic measure, in Harmonic Analysis and Partial Differential Equations (J. Garciá-Cuerra, ed.), Lecture Notes in Math., vol. 1384, Springer-Verlag, 1989.

[S] S. Semmes, A criterion for the boundedness of singular integrals on hypersurfaces, Trans. Amer. Math. Soc. 311 (1989), 501-513.

Department of Mathematics, Rice University, Houston, Texas 77251 